\title{
Manufacturing Learning and Forgetting: Steady State Optimal Batch Size for Constant Demand Case
}

\author{
SunanthaPrime (Teyarachakul)
}

\begin{abstract}
Assuming learning and forgetting in processing units, constant demand rate, and infinite horizon, we analyze costs and properties related to lot sizes in the steady state. Steady State characteristics are described by a convergence in worker experience level or skills. The average per period cost as a function of lot size is found to be non-convex in the steady state. Thus, a simple approach such as first-order condition is not guaranteed to give an optimal solution. We develop sufficient conditions for existence of a uniqueoptimal solution, which are found in some problems. Our study shows that EOQ-type policies that use fixed batch size and produce when inventory reaches zero are not necessarily optimal.
\end{abstract}

Index Terms: manufacturing learning and forgetting, steady state optimal batch size, cost minimization, forms of optimal policy

\section{INTRODUCTION}

You partakein a new production line and you would like to know how many units to produce at any point of time. In result you could just push many items down the process to see what implication it mayhave and just see what materializes. If you attempt to just try and see what happens approach, how does it affect the real cost, and how does itinfluence the production staff learning and forgetting in the long run. So, how do we avoid this potential calamity under manufacturing learning and forgetting and what really are the best lot size and the policy?

In this paper we analyze how to create the optimal fixed batches with learning and forgetting. In the process units given a constant demand rate and infinite problem horizon. This work extends the steady-state results of Teyarachakul, Chand, and Ward [6] and Teyarachakul, Comez, and Tarakci[7] to analyze the cost structure and characteristics, cost-minimization lot size, and forms of the optimal policies. The scope of the problems is what batch production time converges to a single value, as named "single-point convergence" by Teyarachakul, Chand, and Ward [6] and "attractive fixed point" by Teyarachakul, Comez, and Tarakci[7].In other words, the environment under our consideration is when the sequence of experience levels $\left\{G^{\mathrm{k}}(\mathrm{x})\right\}$ converges to a fixed point of experience level $\alpha^{*}$ if the number of lots produced is sufficiently large.This convergence is referred here asthe steady state. We consider the costs characteristic, optimal batch size and policies under this steady state environment.

Revised Manuscript Received on July 20, 2019

Dr. Sunantha Prime (Teyarachakul), Department of Information Systems and Decision Sciences (ISDS) at California State University, Fresno, California, USA.

It was found that cost analysis and optimal policies were not addressed by a number of scholars, who considered similar problems (e.g. Globerson and Levin [2]). Sule [5] and Jaber and Bonney [4] did show the expressions of average costs per unit time; but, Jaber and Bonney's [4] algorithm to obtain the least-cost solution is a heuristic and therefore sub-optimization. Sule[5] assumes the steady-state batch production-time is a linear function of lot size; however, this assumption was proven to be invalid by Elmaghraby [1]. Elmaghraby [1] gives a closed-form expression for the steady-state batch production time given his power forgetting function (the function with a fixed forgetting intercept and a fixed forgetting slope), and he provides cost expressions. However, no discussion on either the shape of average total cost per unit time as a function of lot size or the optimal lot size is given.

Note: The scope of this study is within learning and forgetting in processing units. The subject study does not provide setting up an operations and automatic processing. (See learning and forgetting in setups in Tzur [9] and Teyarachakul, Chand, and Tzur [8])

This paper is structured as following section:

II. PRESENTS COST FORMULATION AND STRUCTURE. THE RESULTS ARE LATER USED TO STUDY THE OPTIMAL FIXED BATCH SIZE IN SECTION 3.

\section{DEVELOPS PROPERTIES OF THE OPTIMAL LOT SIZE.}

IV. DISCUSSES AND PROVIDES RESULTS FOR FORMS OF OPTIMAL POLICIES ON THE EQUAL-SPACED PRODUCTION CYCLES AND CONSTANT BATCH-SIZE.

V. THIS PAPER IS CLOSED BY CONCLUDING REMARK AND THE SUGGESTED POSSIBLE FUTURE RESEARCH.

\section{COST STRUCTURE AND ANALYSIS}

The focus of this section is the derivation of cost expressions and analysis. The optimal fixed batch size is selected based the cost-minimization objective. That is, the optimal fixed batch size is the lot size that minimizes average per period total cost, denoted by ATC(q). It is composed of the costs: (i) average per period setup cost, denoted by $\operatorname{ACk}(\mathrm{q})$; (ii) average per period holding cost, denoted by $\mathrm{ACh}(\mathrm{q})$; and (iii) average per period production labor cost, denoted by $\mathrm{ACp}(\mathrm{q})$. Thus, 


$$
\operatorname{ATC}(q)=\operatorname{ACk}(q)+\operatorname{ACh}(q)+\operatorname{ACp}(q)
$$

Similar to cost expressions in the Economic Order Quantity (EOQ) problems, our $\operatorname{ACk}(q)$ and $\mathrm{ACh}(\mathrm{q})$ are

$\operatorname{ACk}(\mathrm{q})=\frac{S D}{q}$,

$\operatorname{ACh}(\mathrm{q})=\frac{h q}{2}$, where

$S=$ production setup cost (\$ / setup),

$h=$ inventory holding cost ( $\$$ / unit / period), and

$D=$ demand rate (number of units / period)

The above-mentioned cost expressions reveal the mathematics properties of cost structures. Specifically, $\operatorname{ACk}(\mathrm{q})$ is decreasing convex in $\mathrm{q} ; \mathrm{ACh}(\mathrm{q})$ is linearly increasing in q. Expression of $\mathrm{ACh}(\mathrm{q})$ assumes the inventory holding cost charged for both the material and the finished-unit inventories. As part of our cost assumptions, materials are available for the entire batch at the start of production and the same holding cost per kit of materials as per finished-product unit.

Our cost structure differs from the traditional EOQ model in the expression of an average per period direct labor cost, $\operatorname{ACp}(q)$, which is influenced by learning and forgetting. Thus,

$$
\operatorname{ACp}(\mathrm{q})=\frac{w t *(q) D}{q}
$$

where $t^{*}(q)$ is the batch production-time corresponding to experience level $\alpha^{*}(\mathrm{q})$ at the start of production and $\mathrm{w}$ is operator's wage rate (\$ / period). See experience level $\alpha^{*}$ as a converged single-point by Teyarachakul, Chand, and Ward [6]; and attractive fixed pointby Teyarachakul, Comez, and Tarakci [7].

Result 1 shows the possibility of non-convexity of ATC(q) and therefore the first order condition (condition such that $\frac{\partial A T C(q)}{\partial q}=0$ ) could lead to a sub-optimal solution due to multiple local minimums.

RESULT 1: The average per period total cost, ATC(q), could be non-convex.

Proof of Result 1: To prove this result, it is sufficient to provide an example where ATC(q) is non-convex. Example 1 has the following parameters; the first unitproduction time by an inexperienced worker $\mathrm{T}(1)=1$ period, with the use of Wright's learning curve (1936) and learning parameter $\mathrm{m}=$ 0.05 , forgettingparameter $\mathrm{b}=1.2, \mathrm{D}=0.3$ unit $/$ period, $\mathrm{h}=\$$ $0.225 /$ unit / period, $\mathrm{w}=\$ 85.00 /$ period, $\mathrm{S}=\$ 1.00 /$ setup. The corresponding graph of ATC $(\mathrm{q})$ is given in Figure 1; it shows non-convexity. The reason of non-convexity $\operatorname{inATC}(q)$ isnon-convex ACp(q). See Appendix 1 for $\mathrm{ACp}(q)$ characteristics).

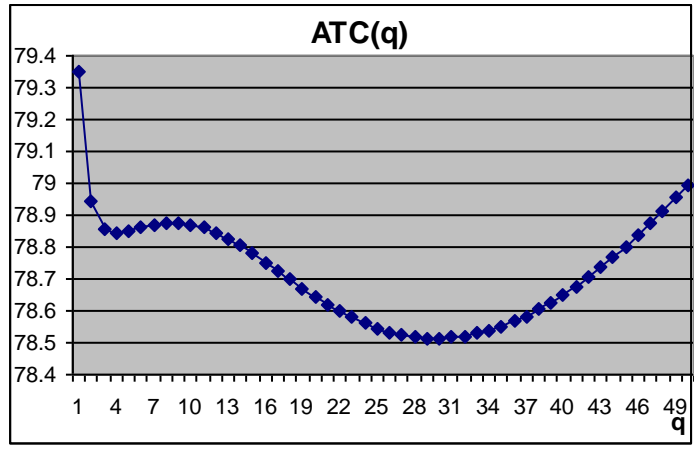

Figure 1. A Counter Example for Convexity of ATC(q).

Based on our simulation runs, $\mathrm{t}^{*}(\mathrm{q})$ appears to be increasing concave (increasing and concave) in q. There is a possibility of such qualification. Lemma 1 considers the effect of $t^{*}(q)$ on $\operatorname{ACp}(q)$ when $t^{*}(q)$ is increasing-concave in q.

Lemma 1: If $\mathrm{t}^{*}(\mathrm{q})$ is increasing-concave in $\mathrm{q}$, then $\mathrm{ACp}(\mathrm{q})$ decreases in $\mathrm{q}$.

Proof of Lemma 1: $\mathrm{ACp}(\mathrm{q})=\frac{w t^{*}(q) D}{q}$ $\frac{\partial A C p(q)}{\partial q}=\frac{w D}{q}\left(\frac{\partial t *(q)}{\partial q}-\frac{t *(q)}{q}\right)$. If $\mathrm{t}^{*}(\mathrm{q})$ is increasing concave in $\mathrm{q}$, then $\frac{t^{*}(q)}{q} \geq \frac{\partial t^{*}(q)}{\partial q}$, and thus $\frac{\partial A C p(q)}{\partial q} \leq 0$.

When we further examine the sign of $\frac{\partial^{2} A C p(q)}{\partial q^{2}}$,there is no clear determined sign due to

$\frac{\partial^{2} A C p(q)}{\partial q^{2}}=\frac{w D}{q}\left(\frac{\partial^{2} t *(q)}{\partial q^{2}}+\frac{2 t *(q)}{q^{2}}-\frac{1}{q} \frac{\partial t *(q)}{\partial q}\right)$, where $\frac{\partial^{2} t *(q)}{\partial q^{2}}$ $\leq 0$, and $\frac{1}{q}\left(\frac{2 t *(q)}{q}-\frac{\partial t *(q)}{\partial q}\right) \geq 0$.

The next Lemma show a sufficient condition for $\mathrm{t} *(\mathrm{q})$ to be increasing-concave.

Lemma 2: If $\alpha *(q)=1, \forall q>q^{\prime \prime}$, then $\mathrm{t}^{*}(\mathrm{q})$ is increasing concave in q for all $\forall q>q^{\prime \prime}$.

Proof of Lemma 2: $\alpha *(q)=1 \forall q>q^{\prime \prime}$

$\Rightarrow \alpha^{*}(\mathrm{q})$ does not depend on $\mathrm{q}, \forall q>q^{\prime \prime}$.

$\Rightarrow$ Replace $\alpha^{*}(\mathrm{q})$ with $\alpha^{*}$.

Recall that $\mathrm{t}^{*}(\mathrm{q})$

$$
\begin{aligned}
& =\frac{T(1)}{1-m}\left((\alpha *+q-0.5)^{1-m}-(\alpha *-0.5)^{1-m}\right) . \\
& \quad \alpha *(q)=1 \forall q>q^{\prime \prime} \\
& \Rightarrow \frac{\partial t^{*}(q)}{\partial q}=T(1)(\alpha *+q-0.5)^{-m} \geq 0 \\
& \Rightarrow \frac{\partial^{2} t^{*}(q)}{\partial q^{2}}=-T(1) m(\alpha *+q-0.5)^{-m-1} \leq 0
\end{aligned}
$$

Thus, $\mathrm{t}^{*}(\mathrm{q})$ is increasing and concave in $\mathrm{q}, \forall q>q^{\prime \prime}$ if $\alpha *(q)=1, \forall q>q^{\prime \prime}$.

$\alpha^{*}(\mathrm{q})=1$ is the scenario in which the interruption interval between 2 successive production batches is sufficiently large to result all learning forgotten prior to the next production run. Next, we will provide results on the optimal lot size. 


\section{THE OPTIMAL FIXED BATCH SIZE Q*}

Results 2 and 3 provide the properties of optimal lot size.

RESULT 2: If $\mathrm{t}^{*}(\mathrm{q})$ is increasing-concave in $\mathrm{q}$, then $\mathrm{q}^{*} \leq$ $\tilde{q}$, where $\tilde{q}$ is the q such that $\frac{h}{2}=\frac{w D t *(q)}{q}+\frac{S D}{q}$. Remark: $\tilde{q}$ is unique because $\frac{h}{2}$ is constant and $\frac{w D t *(q)}{q}+\frac{S D}{q}$ decreases in $\mathrm{q}$.

Proof of Result 2: Recall that

$$
\operatorname{ATC}(\mathrm{q})=\frac{S D}{q}+\frac{h q}{2}+\frac{w t^{*}(q) D}{q} .
$$

We use the concept of marginal cost analysis. That is, by increasing $\mathrm{q}$ by 1 , anincrease in cost is theholding costcomponent going up by $\frac{h}{2}$. The savings consist of (i) the productionlabor cost saving due to worker learning, and (ii) the setup cost per unit decrease. The maximum possible saving is $\operatorname{ACp}(q)+\operatorname{ACk}(q)$. Both $\operatorname{ACp}(q)$ and $\operatorname{ACk}(q)$ (the maximum saving) decrease in $q$ (Lemma 1) while the increase in cost is constant. At $\tilde{q}$, the increase in cost equals the maximumsaving: $\frac{h}{2}=\frac{w D t *(q)}{q}+\frac{S D}{q}$. So, $\forall q>\tilde{q}$,

$\frac{h}{2}>\frac{w D t^{*}(q)}{\tilde{q}}+\frac{S D}{\tilde{q}}$. Equivalently, $\operatorname{ATC}(\mathrm{q})$ increases in $\mathrm{q}$ $\forall q>\tilde{q}$. Thus, $\mathrm{q}^{*} \leq \tilde{q}$.

Result 2 provides an upperbound on $\mathrm{q}^{*}, \tilde{q}$; this limits the range to search for $\mathrm{q}^{*}$. Result 3 establishes a sufficient condition for problems to have a unique optimum.

RESULT 3: If $\frac{\partial^{2} t *(q)}{\partial q^{2}}>\frac{-h}{w D}$ for any q such that $\frac{\partial A T C(q)}{\partial q}=0$, then $\mathrm{q}$ is a unique local minimum, and therefore the global minimum.

Proof of Result 3: Our proof of Result 3 is based on the fact that there is only one minimum if $\frac{\partial^{2} A T C(q)}{\partial q^{2}}>0$ forqsuch that $\frac{\partial A T C(q)}{\partial q}=0$.

To find an expression for $\left.\frac{\partial^{2} A T C(q)}{\partial q^{2}}\right|_{\frac{\partial A T C(q)}{\partial q}=0}$,

$$
\begin{gathered}
\frac{\partial A T C(q)}{\partial q}=w D\left(\frac{q \frac{\partial t^{*}(q)}{\partial q}-t^{*}(q)}{q^{2}}\right)+\frac{h}{2}-\frac{S D}{q^{2}} \\
=\frac{w D}{q}\left(\frac{\partial t^{*}(q)}{\partial q}-\frac{t^{*}(q)}{q}\right)+\frac{h}{2}-\frac{S D}{q^{2}} \\
\frac{\partial A T C(q)}{\partial q}=0 \Leftrightarrow \frac{w D}{q}\left(\frac{\partial t^{*}(q)}{\partial q}-\frac{t^{*}(q)}{q}\right)=\frac{S D}{q^{2}}-\frac{h}{2}
\end{gathered}
$$

$\frac{\partial^{2} A T C(q)}{\partial q^{2}}=\mathrm{wD}$

$\left\{\frac{1}{q}\left(\frac{\partial^{2} t^{*}(q)}{\partial q^{2}}-\left(\frac{q \frac{\partial t^{*}(q)}{\partial q}-t^{*}(q)}{q^{2}}\right)\right)+\left(\frac{\partial t^{*}(q)}{\partial q}-\frac{t^{*}(q)}{q}\right)\left(\frac{-1}{q^{2}}\right)\right\}$

$+\frac{2 S D}{q^{3}}$

$=\frac{1}{q}\left\{w D\left(\frac{\partial^{2} t^{*}(q)}{\partial q^{2}}\right)-\frac{2 w D}{q}\left(\frac{\partial t^{*}(q)}{\partial q}-\frac{t^{*}(q)}{q}\right)\right\}+\frac{2 S D}{q^{3}}$

$\frac{\partial^{2} A T C(q)}{\partial q^{2}}>0 \Leftrightarrow \frac{1}{q}\left\{w D\left(\frac{\partial^{2} t^{*}(q)}{\partial q^{2}}\right)+h\right\}>0$.

A sufficient condition to ensure the unique minimum is $\frac{\partial^{2} t *(q)}{\partial q^{2}}>\frac{-h}{w D}$ for the $\mathrm{q}$ such that $\frac{\partial A T C(q)}{\partial q}=0$.

Example 2. To illustrate Result 3 application, problem parameters: $\mathrm{T}(1)=3.00$ periods $; \mathrm{m}=0.90 ; \mathrm{b}=0.20 ; \mathrm{D}=0.30$ units / period; $\mathrm{h}=\$ 2.00 /$ unit $/$ period; $\mathrm{w}=\$ 35.80 /$ period, $\mathrm{S}=\$ 20.00 /$ setup.

There exist a unique $q$ s.t. $\frac{\partial A T C(q)}{\partial q}=0$.

Let us denote this $q$ as $q^{\prime}$. We found $q^{\prime}=7.282$, and

$$
\begin{aligned}
& \left.\frac{\partial^{2} t^{*}(q)}{\partial q^{2}}\right|_{q^{\prime}=7.282}=-0.106 ; \frac{-h}{w D}=-0.1862 . \\
& -0.106>-0.1862 \text {.Thus, }\left.\frac{\partial^{2} t^{*}(q)}{\partial q^{2}}\right|_{q^{\prime}=7.282}>\frac{-h}{w D} .
\end{aligned}
$$

Thus, this problem satisfies the sufficient condition for the unique minimum of ATC(q) by Result 3; so $\mathrm{q}^{*}$ is 7.282. Graph of ATC $(q)$ is shown in Figure 2.

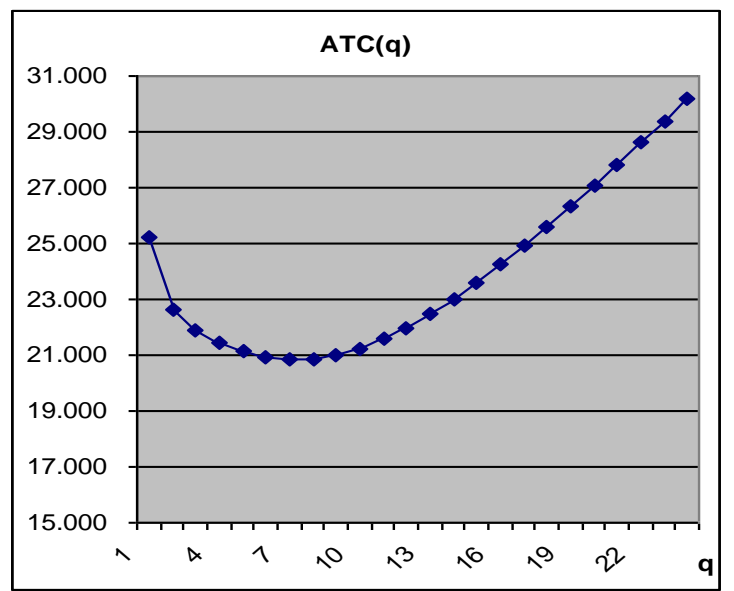

Figure 2 Example of Problem with the Unique Minimum.

The next analysis is to investigate how changes in parameters influence the likelihood of available unique optimum.

Since

$$
\begin{aligned}
& \frac{\partial^{2} t *(q)}{\partial q^{2}}=\frac{\partial\left(\frac{\partial t *(q)}{\partial q}\right)}{\partial q}, \text { and } \\
& \frac{\partial t *(q)}{\partial q}=\frac{S}{w q}-\frac{h}{2 w D} q+\frac{t *(q)}{q} \text { at } \frac{\partial A T C(q)}{\partial q}=0, \text { we get } \\
& \left.\frac{\partial^{2} t *(q)}{\partial q^{2}}\right|_{\frac{\partial A T C(q)}{\partial q}=0}=\frac{\partial\left(\frac{S}{w q}-\frac{h}{2 w D} q+\frac{t *(q)}{q}\right)}{\partial q} \\
& =-\frac{S}{w q^{2}}-\frac{h}{2 w D}+\frac{1}{q^{2}}\left(\frac{\partial t *(q)}{\partial q}-t *(q)\right) \\
& =-\frac{S}{w q^{2}}-\frac{h}{2 w D}+\frac{1}{q^{2}}\left(-t *(q)+\frac{S}{w q}-\frac{h}{2 w D} q+\frac{t *(q)}{q}\right) \\
& =\frac{S}{w q^{2}}\left(-1+\frac{1}{q}\right)-\frac{h}{2 w D}-\frac{h}{2 w D q}+\frac{t *(q)}{q^{2}}\left(-1+\frac{1}{q}\right)<0 . \\
& \text { Recall the condition, }\left.\frac{\partial^{2} t *(q)}{\partial q^{2}}\right|_{\frac{\partial A T C(q)}{\partial q}=0}>\frac{-h}{w D}, \text { for the }
\end{aligned}
$$

existence of the unique optimum.

$$
\left.\frac{\partial^{2} t *(q)}{\partial q^{2}}\right|_{\frac{\partial A T C(q)}{\partial q}=0}>\frac{-h}{w D}
$$


$\Leftrightarrow \frac{S}{w q^{2}}\left(-1+\frac{1}{q}\right)-\frac{h}{2 w D q}+\frac{t(q)}{q^{2}}\left(-1+\frac{1}{q}\right)>-\frac{h}{2 w D}$.

Thus, the likelihood of the unique minimum to exist is increasing with

(i) $\mathrm{h}$ increases and / or

(ii) $\mathrm{S}$ and / or D decrease.

That is, increases in $\mathrm{h}$ and / or decreases in $\mathrm{S}$ and / or D smooth out the local minimums, and results in fewer numbers of local minimums.

We have analyzed the average total cost per period and found that it could be non-convex. Then, we provided an upper bound on $\mathrm{q}^{*}$ and a sufficient condition for the existence of unique optimum in some problems. In the next section, forms of optimal policies will be studied.

\section{FORMS OF OPTIMAL POLICIES}

Under EOQ model, an optimal batch sizing policy satisfies the specifications of (i) a constant batch size, and (ii) production begins when inventory hits zero. Such policy is not held optimal in the manufacturing environment withmanufacturing learning and forgetting as evidenced by counter examples. That is it's more cost efficient for some production runs to begin when inventory is positive (early production) rather than waiting until all units of inventory are depleted.

In our literature review,Jaber and Bonney [4] and Elmaghraby [1] allowed lot sizes to be unequal; however, they provided no discussion on whether unequal lot sizes could improve costs as compared to a fixed lot size. Also, lot sizes in numerical examples of Jaber and Bonney [4] converge to a constant lot size; but, their algorithm is heuristic, so there is no guarantee that the algorithm converges to the optimal fixed batch size. The study in this section contributes to the work in this area by exploring whether the "traditional EOQ-type" properties remain optimal under manufacturing learning and forgetting.

\section{A.Policy on Equal-Spaced Production Cycles}

RESULT 4: Given restrictions of a constant batch size and equal-spaced production cycles, the optimal policy is to have production runs start when inventory reaches zero (Zero Inventory Policy or ZIP).

Proof of Result 4: Given equal-spaced production cycles, the cycle length of $\frac{q}{D}$. With the constant cycle length of $\frac{q}{D}$, for any given an experience level $\alpha$, the amount of learning $\ell(\alpha)$ and the amount of forgetting $f(\alpha)$ under ZIP are the same as those under non-ZIP. Hence, both $\alpha^{*}(\mathrm{q})$ and $\mathrm{t}^{*}(\mathrm{q})$ are the same under these 2 different policies. By starting the production runs when the inventory is positive, we end up with an extra inventory holding cost.

Result 5 is when the restriction of equal-spaced production runs is relaxed.

RESULT 5: Given a fixed batch size of q, unequal-spaced production cycles may improve average total cost per period.
Proof of Result 5. Counter examples to the optimal policy of EOQ is used to support the result.

Consider 2 different policies in steady state, in which the sequence of experience level $\left\{\alpha_{n}\right\}$ converges to $\left.\alpha^{*}(q)\right)$.

Policy 1 is to have production cycles of equal lengths and to place orders only when inventory reaches zero; under this policy, the production repeats itself in every production cycle with experience level $\alpha^{*}(q)$ at the start of each cycle. (See Figure 3.)

Policy 2 is to use a fixed $\mathrm{q}$ (the same as q in Policy 1 ), but to have production cycles unequal in length in some cycles, which implies production taking place before inventory reaches zero in some cycles. To illustrate Policy 2, we select an example in which production repeats itself every 2 cycles; that is, the $1^{\text {st }}$ and the $2^{\text {nd }}$ production runs of each repeated cycle start with experience levels $\alpha_{1}\left(\mathrm{q}\right.$, Inv) and $\alpha_{2}(\mathrm{q}$, Inv), respectively, where Inv is inventory level when the $2^{\text {nd }}$ production run in the repeated cycle begins and Inv $>0$. Note that $\alpha_{1}\left(\mathrm{q}\right.$, Inv) and $\alpha_{2}(\mathrm{q}$, Inv) are steady-state experience levels under Policy 2. (See Figure 4.)

Expressions for batch production-times, experience levels when the production starts and costs are given in Appendix 2.

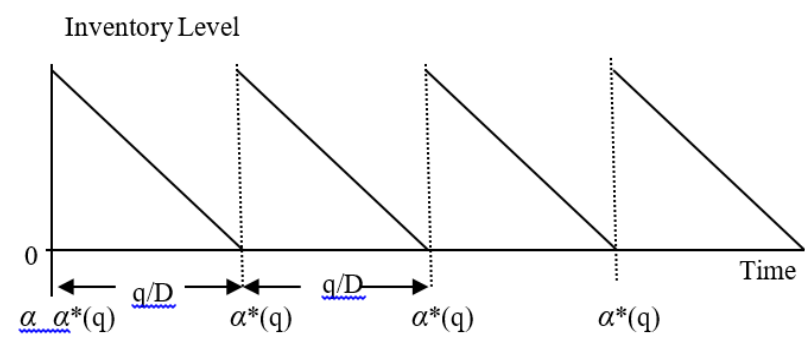

Figure 3. Policy 1: Equal-Spaced Production Cycles and ZIP

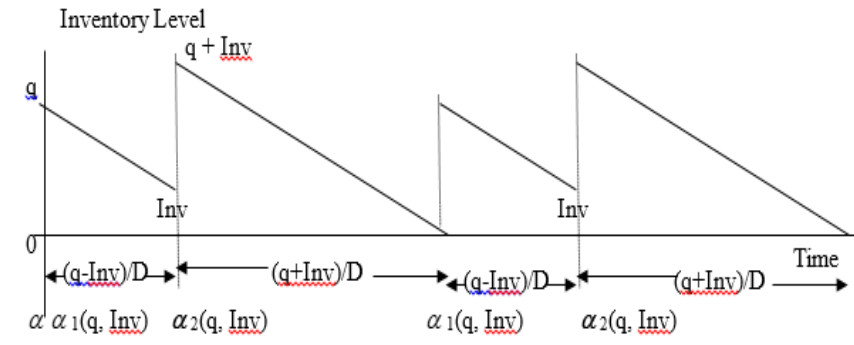

Figure 4. Policy 2: Unequal-Spaced Production Cycles

To compare the performance under these policies, Example 3 is used and cost corresponding to example 3 is provided in Table 1. Example 3 with problem parameters: $\mathrm{T}(1)=1.00$ periods $; \mathrm{m}=0.05 ; \mathrm{b}=1.20 ; \mathrm{D}=1.00$ units / period; $\mathrm{h}=\$ 0.33 /$ unit $/$ period; $\mathrm{w}=\$ 150 /$ period, $\mathrm{S}=\$ 3 /$ setup, and $q=40$ units $/$ batch. 
Table-I Average Cost per Period under Policies 1 and 2

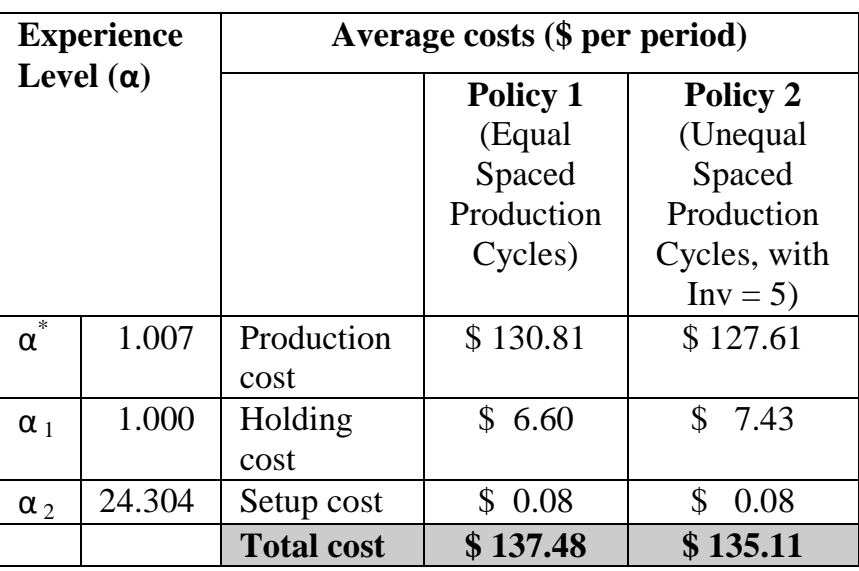

We have shown that the optimal "traditional EOQ-type" policies may not be optimal in our problemsetting. We will close our study in Section 5 by providing concluding remark and future research directions.

\section{V.CONCLUDING REMARK}

An optimal fixed batch size minimizes the average per period cost of holding inventory, setting up machine(s) or tool(s) and direct labor when production reaches a steady state. We consider examples when the batch production-time converges to a unique value in the steady state. We found that the average labor cost per period could be non-convex due to learning and forgetting. Thus, the average total cost per period could be non-convex. We specify sufficient conditions for a unique minimum in certain cases. Unlike the "EOQ-type" optimal policy, the following policies: (i) production starts only when inventory hits zero, and (ii) production cycles are equal spaced, do not necessarily hold at optimality.

An interesting extension is further analysis of cost structures and forms of optimal policies in the case when batch production-time converges to a unique value. Specific extension is to develop an algorithm to find a fixed optimal batch size, and specifications of the form of optimal policies. For example, since the average total cost could be improved if the production starts before inventory reaches zero in some cycles, a natural question would be what the optimal positive inventory is when productions begin in those cycles.

\section{APPENDIX1}

$\operatorname{ACp}(q)$ is Neither Concave nor Convex

Table 2 together with Figure 5 shows a counter example to concave and/or convex average per period production labor cost $\operatorname{ACp}(q)$.

\begin{tabular}{|c|c|c|c|c|c|}
\hline $\mathbf{T}(\mathbf{1})$ & 3.00 & $\mathbf{q}$ & $\boldsymbol{\alpha}^{*}(\mathbf{q})$ & $\mathbf{t}^{*}(\mathbf{q})$ & $\mathbf{A C p}(\mathbf{q})$ \\
\cline { 2 - 5 } $\mathbf{m}$ & 0.90 & $\mathbf{1}$ & 1.930 & 1.693 & 18.186 \\
$\mathbf{b}$ & 0.20 & $\mathbf{2}$ & 1.610 & 3.289 & 17.664 \\
$\mathbf{D}$ & 0.30 & $\mathbf{3}$ & 1.388 & 4.716 & 16.884 \\
\cline { 1 - 2 } $\mathbf{h}$ & $\$ 2.00$ & $\mathbf{4}$ & 1.241 & 5.938 & 15.943 \\
$\mathbf{w}$ & $\$ 35.80$ & $\mathbf{5}$ & 1.146 & 6.952 & 14.934 \\
$\mathbf{S}$ & $\$ 20.00$ & $\mathbf{6}$ & 1.086 & 7.782 & 13.930 \\
& & $\mathbf{7}$ & 1.050 & 8.460 & 12.981 \\
& & $\mathbf{8}$ & 1.029 & 9.023 & 12.113 \\
& & $\mathbf{9}$ & 1.016 & 9.500 & 11.336 \\
& & $\mathbf{1 0}$ & 1.009 & 9.914 & 10.648 \\
& & $\mathbf{1 1}$ & 1.005 & 10.282 & 10.039 \\
& & $\mathbf{1 2}$ & 1.003 & 10.614 & 9.500 \\
& & $\mathbf{1 3}$ & 1.002 & 10.919 & 9.021 \\
& & $\mathbf{1 4}$ & 1.001 & 11.202 & 8.594 \\
& & $\mathbf{1 5}$ & 1.000 & 11.466 & 8.210 \\
& & $\mathbf{1 6}$ & 1.000 & 11.715 & 7.864 \\
\hline
\end{tabular}

Table 2.An Example with Calculated Average per Period Production Labor CostACp(q).

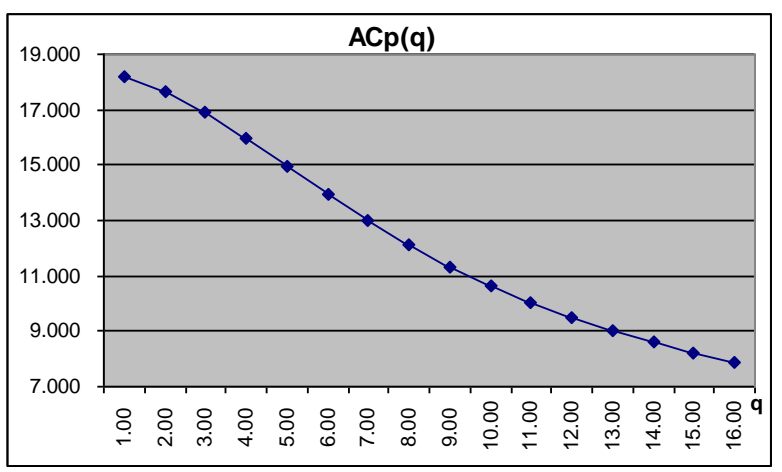

Figure 5. A Counter Example for Convex and/or Concave $\operatorname{ATC}(q)$

\section{APPENDIX 2}

Unequal-Spaced Production Cycles

\subsection{Calculation of $\alpha^{*}(q), \alpha_{i}\left(\alpha_{i-1(i+1)}, q\right.$, Inv $)$ and $t^{*}(q)$ and $\mathbf{t}_{\mathbf{i}}(\mathbf{q})$}

We denote $t_{i}(q)$ and $t^{*}(q)$ as the batch production time corresponding to $\alpha_{i}\left(\alpha_{i-1(i+1)}, q\right)$ and $\alpha^{*}(q)$,respectively. $\alpha^{*}(\mathrm{q})=\left\{1-\left[1-(\alpha *(q)+q)^{-m}\right] \exp \left[-b\left(\frac{q}{D}-t *(q)\right)\right]\right\}^{\frac{-1}{m}}$ $\alpha_{1}(\mathrm{q}$, Inv)

$=\left\{1-\left[1-\left(\alpha_{2}(q, \operatorname{Inv})+q\right)^{-m}\right] \exp \left[-b\left(\frac{q+\operatorname{Inv}}{D}-t_{2}(q)\right)\right]\right\}^{\frac{-1}{m}}$

$\alpha_{2}(\mathrm{q}, \operatorname{Inv})$

$=\left\{1-\left[1-\left(\alpha_{1}(\mathrm{q}, \text { Inv })+q\right)^{-m}\right] \exp \left[-b\left(\frac{q-I n v}{D}-t_{2}(q)\right)\right]\right\}^{\frac{-1}{m}}$

$\mathrm{t}_{\mathrm{i}}(\mathrm{q})$

$=\frac{a}{1-m}\left\{\left(\alpha_{i}(q, \operatorname{Inv})+q-0.5\right)^{1-m}-\left(\alpha_{i}(q, \operatorname{In} v)-0.5\right)^{1-m}\right\}$

$\mathrm{t}^{*}(\mathrm{q})=\frac{a}{1-m}\left\{(\alpha *(q)+q-0.5)^{1-m}-(\alpha *(q)-0.5)^{1-m}\right\}$

\subsection{Cost Calculation}

Policy 1 : Equal-spaced production cycles and placing order when inventory hits zero. 
Production cost $/$ period $=\frac{w t *(q) D}{q}$

Holding cost / period $=\frac{h}{2} \mathrm{q}$

Policy 2 : Unequal-spaced production cycles

Production cost $/$ period $=\left(\mathrm{t}_{1}(\mathrm{q})+\mathrm{t}_{2}(\mathrm{q})\right) \frac{w D}{2 q}$

Holding cost / period

$$
=\frac{h}{4 q}\left[(q-\operatorname{In} v)(q+\operatorname{Inv})+(q+\operatorname{In} v)^{2}\right]
$$

\section{REFERENCES}

1. S. E. Elmaghraby, "Economic manufacturing quantities under condition of learning and forgetting",Production Management and Control, Vol 1 1990, pp. 196-208.

2. S. Globerson, and N. Levin, "Technical paper: Incorporating forgetting into learning curves", International Journal of Operations and Production Management, Vol 7, 1987, pp. 80-94.

3. M. Y. Jaber, and M. Bonney, "Production breaks and the learning curve: The forgetting phenomenon",Applied Mathematics Modelling, Vol 20,1996, pp. 162-169.

4. M. Y. Jaber, and M. Bonney, "The effects of learning and forgetting on the optimal lot size quantity of intermittent production runs",Production Planning and Control, Vol 9, 1998, pp. 20-27.

5. D. R. Sule, "The effect of alternate periods of learning and forgetting on economic manufacturing quantity",AIIE Transactions, Vol 10, 1978, pp. 338-343.

6. S. Teyarachakul, S. Chand, and J. Ward, "Batch sizing under learning and forgetting: Steady state results for the constant demand case",Operations Research Letters, Vol. 36, 2008, pp. 589-593.

7. S. Teyarachakul, C. Comez, and H. Tarakci, "Steady-state skill levels of workers in learning and forgetting environments: A dynamical system analysis", European Journal of Operational Research, Vol. 232, 2014, pp. 9-21.

8. S. Teyarachakul, S. Chand, and M. Tzur, "Lot sizing with learning and forgetting in setups: analytical results and insights",Naval Research Logistics, vol. 63(2), 2016, pp. 93-108.

9. M. Tzur, "Learning in setups: analysis, minimal forecast horizons and algorithms",ManagementScience, Vol. 42, 1996, pp. 1732-1743.

\section{AUTHORS PROFILE}

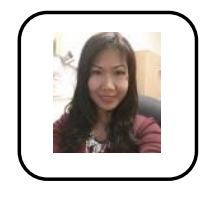

Dr. Sunantha Prime (Teyarachakul)She is an Associate Professor for the Decision Sciences in the Department of Information Systems and Decision Sciences (ISDS) at California State University, Fresno, California, USA (2015-present). She was a tenured Associate Professor of Management at MacEwan University in Edmonton, Alberta, Canada (2012-2015). She also taught at ESSEC Business School in Paris, France (2008-2011), and at Minnesota State University Moorhead (2003-2008). Dr. Prime has been very active in academic research and co-authored more than 10 journal articles in Naval Research Logistics (NRL), European Journal of Operational Research (EJOR), IIE Transactions, Production and Operations Management (POMS), Operations Research Letters, Journal of Computer Information Systems, and Journal of Business Administration.. She has a book chapter published in "Learning Curves: Theory, Models and Applications, CRC Press, 2011. Dr. Prime was awarded a Ph.D. in Operations Management at the Purdue University, Indiana, USA. She received an MBA from the University of North Carolina at Chapel-Hill, North Carolina, USA. 